\title{
NUMERICAL SIMULATION OF FATIGUE FRACTURE OF THE TURBINE DISC
}

\author{
Lucjan Witek \\ Rzeszów University of Technology, Rzeszów, Poland
}

\begin{abstract}
This paper presents the results of the crack propagation analysis of an aircraft engine turbine disc. In the first part of the work the finite element method was used for calculation of the stress state and the stress intensity factor (SIF, $K_{I}, K$-factor) in the turbine disc with an embedded quarter-elliptical corner crack, subjected to low-cycle thermo-mechanical fatigue. To refine the Kfactor calculation, specially degenerated finite elements were used. These elements provide stress singularity suitable for the linear-elastic material of the disc. The performed calculations yielded the stress intensity factor $K_{I}$ for different crack sizes. Subsequently, $\Delta K$ parameter was determined as a difference of the $K_{I}$ values calculated for the turbine's speeds equal to 6373 and 14200 RPM. Based on the Paris-Erdogan equation and the obtained $\Delta K$ values, the fatigue crack growth plot for the turbine disc subjected to complex thermo-mechanical loads was determined.
\end{abstract}

\section{INTRODUCTION}

The service life of critical aerospace components is governed by the modes of degradation and failure such as: fatigue, fracture, yielding, creep, corrosion, erosion, wear, etc. Gas turbine discs are usually the critical engine components, which must endure substantial mechanical and thermal loading. If a problem arises in the turbine section it will significantly affect the whole engine's performance and, obviously, safety of an aircraft. A broken blade can be contained within the engine casing while a catastrophic failure of the turbine wheel could cause puncture of the engine casing by the larger fragments of the disc. Turbine discs have three critical regions on which the attention should be focused: the dovetail-rim area (fir-tree slots, serration fitting), the assembly holes and the hub zone. The loads associated with these regions are mainly the centrifugal forces and thermal stresses. The joint between the turbine blade and the disc usually represents the most sensitive area from the point of view of static and fatigue strength.

The stress and failure analysis of the jet engine turbine has been the focus of a number of investigations. The problem of numerical evaluation of the stress state in the dovetail-rim area of the disc and blades was described by Chan et al. [1], Masataka [2], Meguid et al. [3], Papanikos et al. [4] and Zboinski [5]. Of interest is also the work by McEvily [6], in which the author analyzed the failures of the engines used to power the MD-88, DC-10 and B-737 planes. In these cases, the problem of unexpected fatigue failure of the turbine was the reason behind many aircraft crashes.

The aero-engine components are subjected to thermo-mechanical loads. Moreover, the geometry of the turbine blade or turbine disc is complex. For these reasons the crack propagation analysis in the rotational engine components is not widely described in literature. The crack propagation analysis or the stress intensity factor calculation for the turbine components was described by Barlow et al. [7], Slavik et al. [8], Shuang [9] and Shlyannikov et all [10]. The results of the numerical and experimental crack initiation, crack propagation and fatigue life analysis of the rotational engine components were also described in the works [11-17]. This publication is 
a continuation of the work [11], which investigated fatigue fracture of the turbine disc of the aircraft engine (Fig. 1).

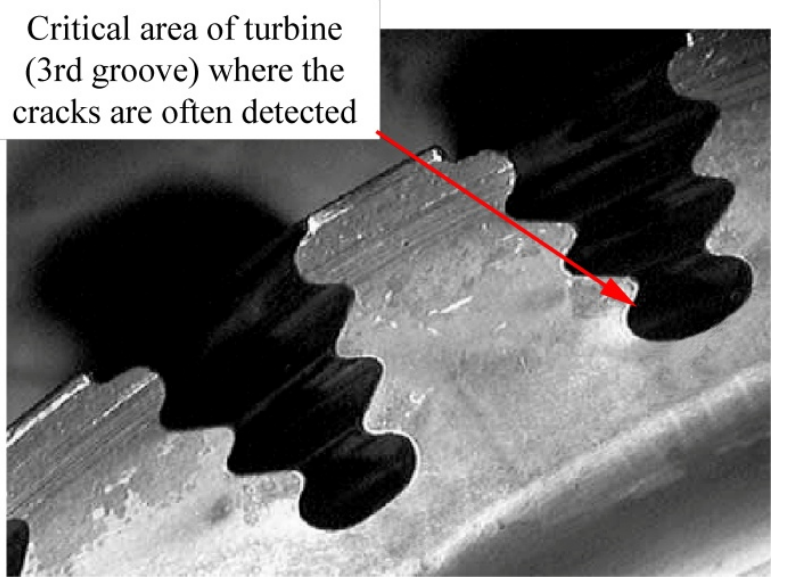

Fig. 1. Fir-tree slots of the disc where fatigue cracks were often detected during engine operation.

The main research goal of this study was to estimate the stress intensity factor in the quarterelliptical crack, embedded in the serration area of the turbine disc. Additionally, the work aimed at the estimation of crack growth dynamics in the turbine disc subjected to low-cycle thermomechanical fatigue caused by fluctuation of the rotational speed of the engine rotor.

The estimation of the stress intensity factor and the crack growth rate for the turbine disc subjected to thermo-mechanical loads is an original research achievement in the area of fracture mechanics. The results of this work have also great practical significance. Crack growth dynamics is very important for operation of aircraft according to the "damage tolerance" methodology. This method assumes that in correctly defined technical services a fatigue crack should be detected before it grows to the critical size. The critical size of a crack means that the stress intensity factor in the crack achieves the critical value $\mathrm{K}_{\mathrm{c}}^{\mathrm{I}}$. When the $\mathrm{K}$-factor in the crack is close to $\mathrm{K}_{\mathrm{c}}^{\mathrm{I}}$, then the fatigue process is unstable and the turbine disc can burst rapidly.

\section{STRESS ANALYSIS OF THE TURBINE DISC WITHOUT DEFECTS.}

In the first part of the work, a detailed geometrical model of the 1/89 turbine segment was created. The border surfaces of the disc used during the parametric $3 \mathrm{D}$ model definition are presented in Fig. 2a. The cyclic symmetry option with the appropriate boundary conditions enabled modeling only a small part of the disc with one blade.

The finite element program MSC-Patran [18] was used to create both the geometry and discrete models of the disc segment and the blade. The finite element model of turbine presented in Fig. $2 \mathrm{~b}$. consists of about 23608 solid elements. To model the mechanical interface of the adjacent surfaces of the disc and the blade, the "master-slave" type of contact [19] with the friction coefficient of 0.1 was defined. 

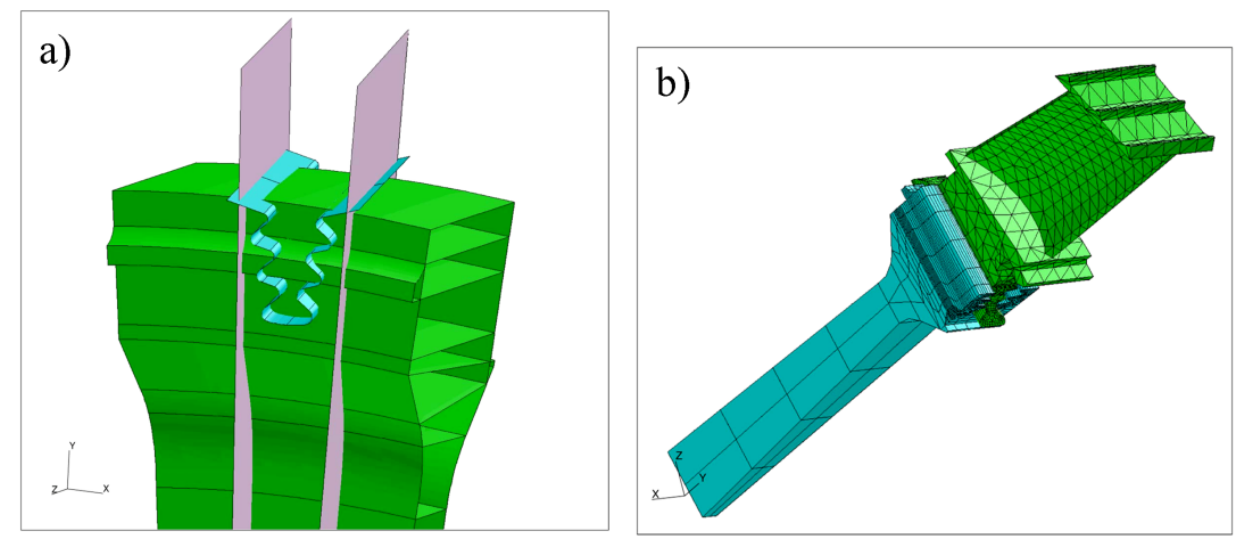

Fig. 2. Border surfaces used to create of the geometrical model of the disc (a) and the discrete model of the turbine segment used in computations (b).

A rotating hot section component in a turbine engine is in general subjected to a combination of surface (aerodynamic), centrifugal and thermal loads. Surface loads are associated with aerodynamic forces resulting mainly from the impingement of hot gases on the blade surfaces. The centrifugal loads arising from the mass of the rotated disc and the blades are usually the critical loads acting on the turbine disc. This load was determined through finite element calculation after defining the axis of symmetry, the rotational rotor speed and also the disc and the blade material density. In this analysis, the operational turbine speed of 14200 RPM (rotation per minute) was applied. While the engine works, a non-uniform temperature field arises in the turbine disc and the blade. The thermal load used in the computations is presented in Fig. 3a.

a)

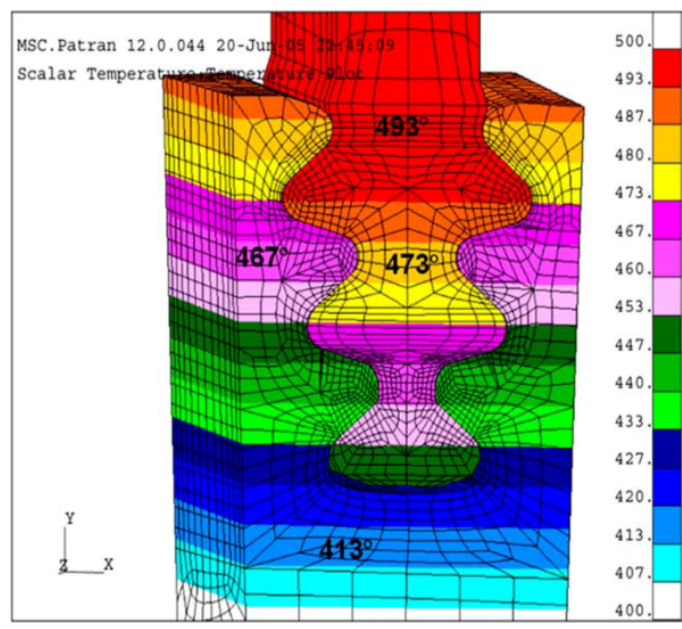

b)

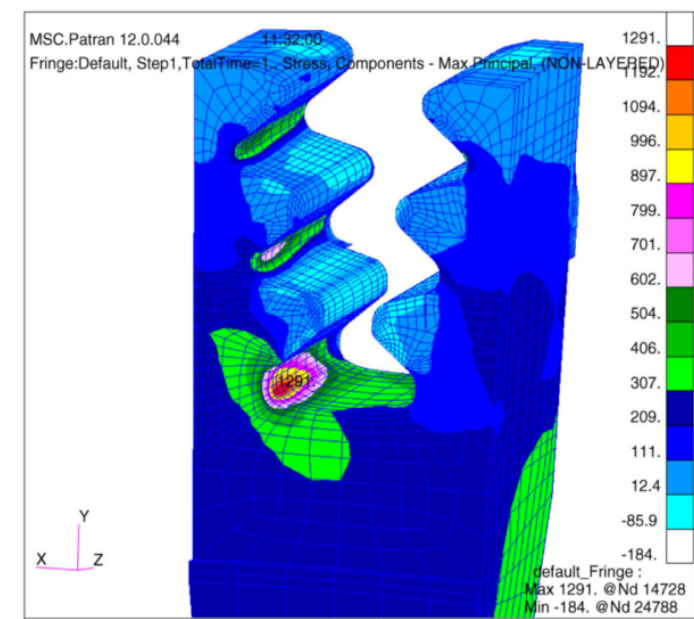

Fig. 3. The thermal field defined for the models of the disc and the blade in the dovetail-rim region (Celsius scale) (a) and the maximum principal ( $\left.\sigma_{1}\right)$ stress distribution in the turbine disc subjected to thermo-mechanical loads (b).

Fig. 3b. shows that the area of the maximum principal stress $(1291 \mathrm{MPa})$ is located on the corner of the $3^{\text {rd }}$ lower slot of the disc. The maximum principal stress is particularly interesting from the point of view of fatigue strength because it is tensile stresses that contribute the most to the fatigue fracture. 


\section{STRESS ANALYSIS OF THE TURBINE DISC WITH A QUARTER-ELLIPTICAL CRACK}

To refine the K-factor calculation (according to linear-elastic fracture mechanics), specially degenerated finite elements were used to assure the type $1 / \mathrm{J}_{\mathrm{r}}$ stress singularity around the crack tip [20]. The crack tip should be first modeled as a ring with the finite radius (Fig. 4a.). Next, the nodes $\mathrm{a}, \mathrm{b}$ and $\mathrm{c}$ should be collapsed into one point (the crack tip). These degenerated elements provide $1 / \mathrm{r}$ singularity, suitable for perfectly plastic materials. For linear-elastic problems, it is necessary to create square $(1 / \sqrt{ } \mathrm{r})$ singularity by moving the midside nodes to the $1 / 4$ parametric position (Fig. 4b.).

Preliminary stress analysis described in Chapter 2 showed that in the disc without defects the maximum principal stress occurs in the third slot of the serration fitting. In the presented simulation it was assumed that the crack would propagate from the zone of the disc where the maximum tension stress $\left(\sigma_{1}\right)$ was observed (the third slot of the serration fitting). Based on the results presented in work [11] (the shape of real fatigue beach marks), the quarter-elliptical crack was defined in the numerical model (Fig. 5a). This figure presents three layers of finite elements located around the crack tip. These layers are natural border surfaces of contours integral during the stress intensity factor calculations.

In the present analysis, the numerical models with the following crack lengths were created: $1 \mathrm{~mm} ; 1,5 \mathrm{~mm} ; 2,5 \mathrm{~mm} ; 4 \mathrm{~mm}$ and $7 \mathrm{~mm}$ (Fig. 2a.). In order to define the crack front position (on $\mathrm{K}$ or $\Delta \mathrm{K}$ plots), the curvilinear parametric coordinate system was defined (Fig. 5a.).

a)

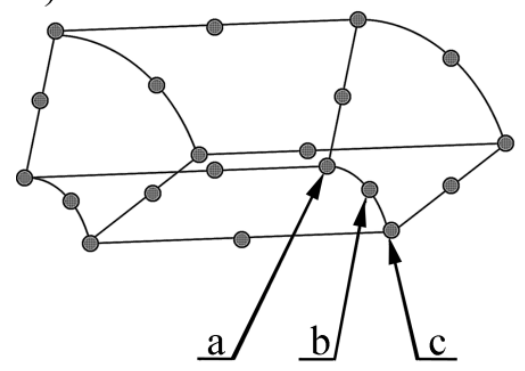

b)

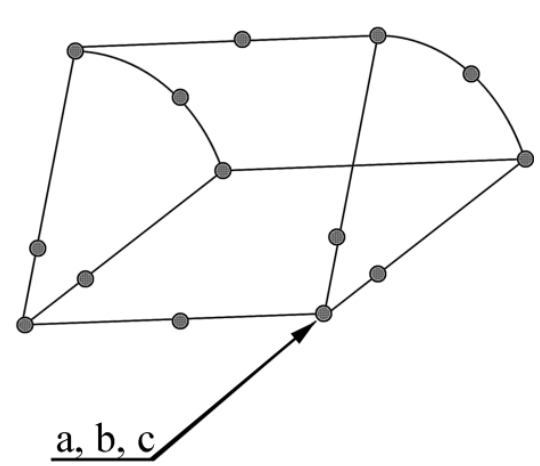

Fig. 4. Typical (a) and degenerated HEX-20 finite element assuring $(1 / \sqrt{ } r)$ singularityused in linear-elastic fracture mechanics.

a)

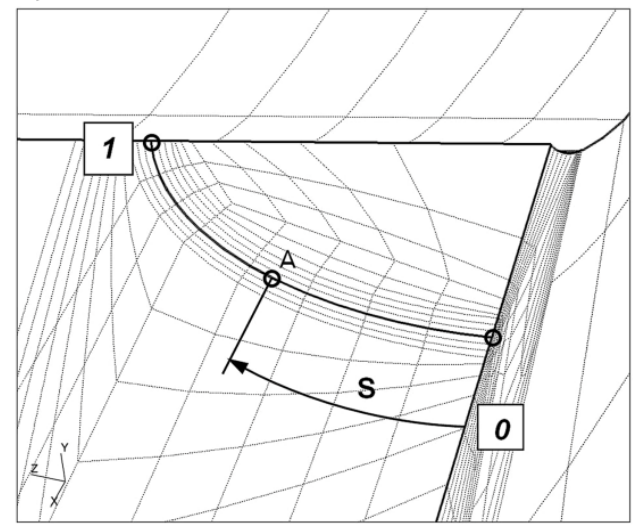

b)

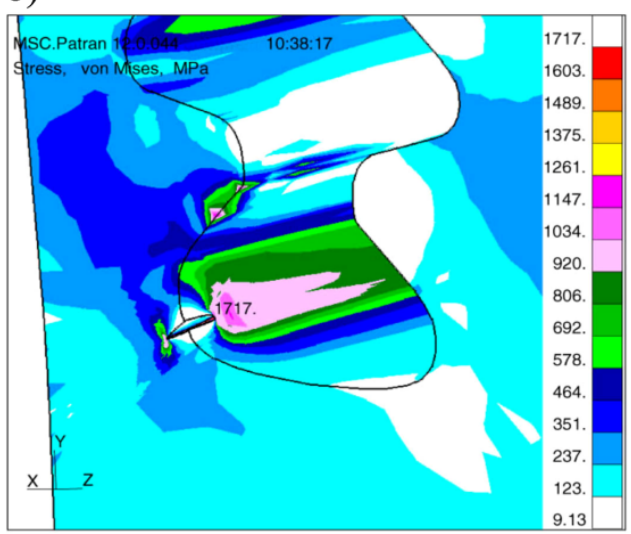

Fig. 5. Shape of the crack front with the defined curvilinear coordinate system used in visualization of $K_{I}$ values (a) and the von Mises stress distribution on the surface of the disc with an embedded short crack. 
As a result of FEM calculations the stress fields for the disc with cracks were obtained. Figure $5 \mathrm{~b}$ shows that the maximum value of the von Mises stress in the region of the crack tip is about $1700 \mathrm{MPa}$ (for the crack length $\mathrm{a}=1 \mathrm{~mm}$ and the turbine's operational speed $\mathrm{n}=14200 \mathrm{RPM}$ ). For a better visualization of the crack opening, the scale of the deformation in Fig. 5b. was increased 10 times.

\section{THE STRESS INTENSITY FACTOR CALCULATIONS FOR THE CRACKS EMBEDDED IN THE TURBINE DISC}

One of the main parameters used in fracture mechanics is the stress intensity factor (SIF, K, Kfactor). The $\mathrm{K}$-factor is an important parameter for definition of the stress state around the crack tip. SIF is also needed to determine the crack growth dynamics. The values of SIF for simple structures can be computed using analytical methods. Nevertheless, for the complex geometry structures (e.g. the turbine disc), the analytical solutions for the K-factor estimation are not known.

As a result of the performed calculations the stress intensity factor $K_{I}$ for different crack sizes (in the range of 1-7 mm) was calculated. Values $\mathrm{K}_{\mathrm{I}}$ for the second contour integral are presented in Fig. 6. The obtained results allowed determining the $\Delta \mathrm{K}$ parameter as a difference between the $\mathrm{K}_{\mathrm{I}}$ values calculated for the turbine speed equal to 6373 and 14200 RPM (Tab. 1, Fig. 7).

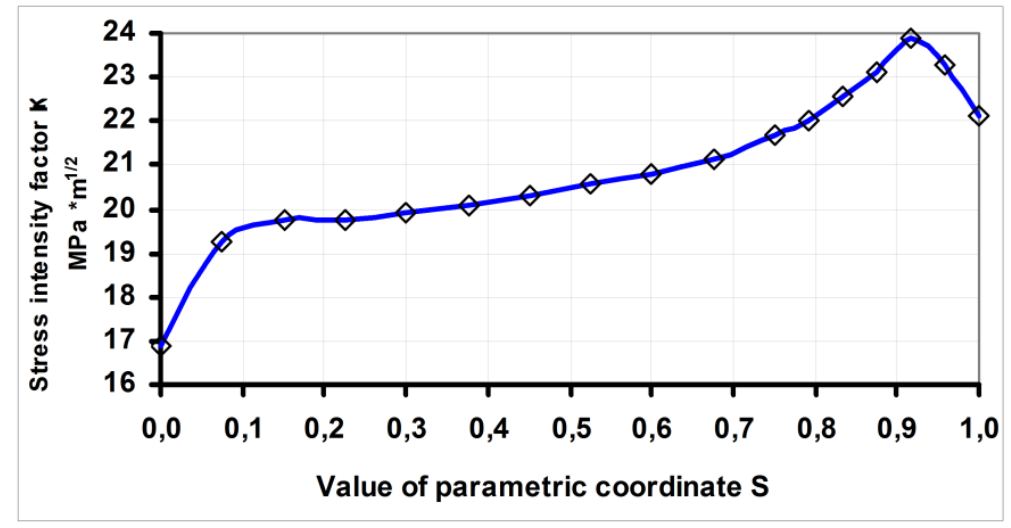

Fig. 6. Value of the stress intensity factor $\left(K_{D}\right)$ computed from the $2^{\text {nd }}$ contour integral for a short crack $(a=1 \mathrm{~mm}, n=14200 \mathrm{RPM})$.

Tab. 1. Values of $\triangle K$ for different crack sizes for the disc speed range of $6373-14200 R P M$.

\begin{tabular}{|c|c|c|c|c|c|}
\hline \multirow{2}{*}{$\begin{array}{c}\text { Value of } \\
\text { coordinate } \mathbf{S}\end{array}$} & \multicolumn{5}{|c|}{ delta $\mathbf{K}=\mathbf{K}_{\mathbf{m a x}}-\mathbf{K}_{\mathbf{m i n}}\left[\mathbf{M P a}{ }^{*} \mathbf{m} \mathbf{1 / 2}\right.$ ] for $\mathbf{a =}$} \\
\cline { 2 - 6 } & $\mathbf{1} \mathbf{~} \mathbf{m m}$ & $\mathbf{1 . 5} \mathbf{~ m m}$ & $\mathbf{2 , 5} \mathbf{~ m m}$ & $\mathbf{4} \mathbf{m m}$ & $\mathbf{7 m m}$ \\
\hline 0,000 & 16,45 & 18,99 & 22,79 & 28,48 & 37,98 \\
\hline 0,075 & 18,73 & 21,32 & 25,59 & 31,99 & 42,65 \\
\hline 0,150 & 19,24 & 21,74 & 26,09 & 32,61 & 43,48 \\
\hline 0,225 & 19,21 & 21,82 & 26,19 & 32,74 & 43,65 \\
\hline 0,300 & 19,39 & 22,09 & 26,51 & 33,14 & 44,18 \\
\hline 0,375 & 19,56 & 22,34 & 26,80 & 33,51 & 44,67 \\
\hline 0,451 & 19,78 & 22,60 & 27,12 & 33,89 & 45,19 \\
\hline 0,526 & 20,01 & 22,89 & 27,47 & 34,33 & 45,78 \\
\hline 0,601 & 20,26 & 23,18 & 27,82 & 34,78 & 46,37 \\
\hline 0,676 & 20,59 & 23,58 & 28,29 & 35,36 & 47,15 \\
\hline 0,751 & 21,11 & 24,20 & 29,04 & 36,30 & 48,40 \\
\hline 0,792 & 21,45 & 24,69 & 29,62 & 37,03 & 49,37 \\
\hline 0,834 & 21,98 & 25,39 & 30,47 & 38,08 & 50,78 \\
\hline 0,875 & 22,49 & 26,16 & 31,39 & 39,24 & 52,32 \\
\hline 0,917 & 23,27 & 27,53 & 33,04 & 41,30 & 55,07 \\
\hline 0,958 & 22,68 & 27,38 & 32,85 & 41,06 & 54,75 \\
\hline 1,000 & 21,56 & 25,32 & 30,39 & 37,99 & 50,65 \\
\hline
\end{tabular}




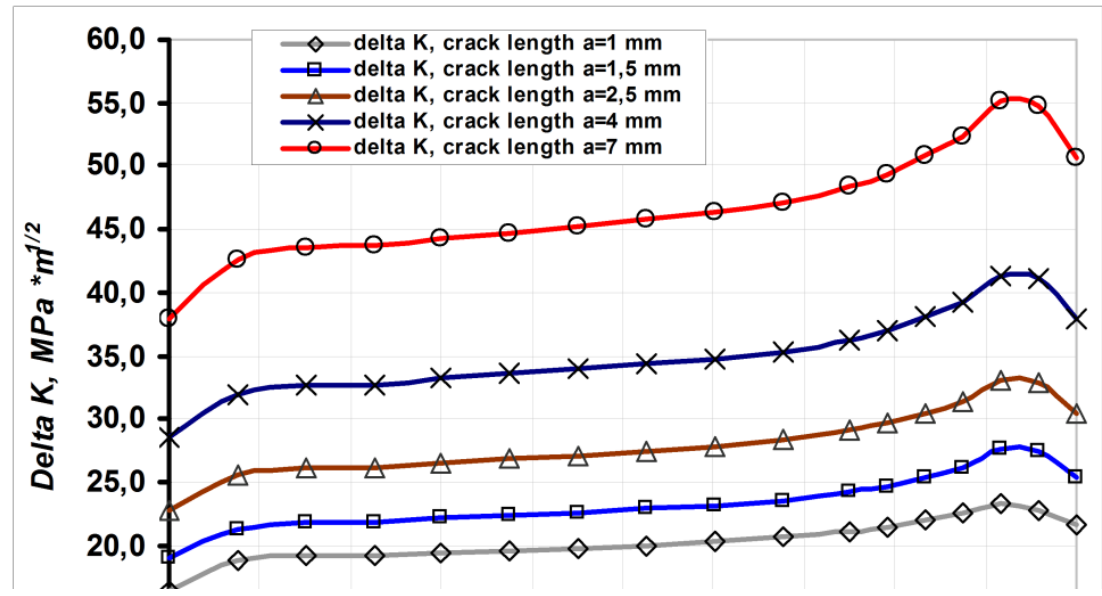

Fig. 7. Values of $\Delta K$ for crack sizes between 1 and $7 \mathrm{~mm}$, for the disc speed range of $6373-14200$ RPM.

\section{CRACK GROWTH ANALYSIS}

The stress intensity factor is the main parameter in crack growth analysis. According to the Paris-Erdogan equation [20], the crack growth rate can be computed from the following formula:

$$
\frac{d a}{d N}=C \cdot(\Delta K)^{m}
$$

where:

$d a / d N$ - the crack growth rate (growth of the crack after one fatigue cycle)

$C, m \quad$ - the Paris constants

Value of $\Delta \mathrm{K}$ is computed from the equation:

where:

$$
\Delta K=K_{\max }-K_{\min }
$$

$K_{\max }$ - maximum value of the K-factor computed for the disc speed n=14200 RPM

$K_{\min }$ - minimum value of the K-factor computed for the disc speed n=6373 RPM

$\mathrm{C}$ and $\mathrm{m}$ values were taken from the da/dN curve, obtained for the disc material tested in $650{ }^{\circ} \mathrm{C}$ (for specimens cut from the forged turbine disc) [21]. For the curve obtained from test $20 \mathrm{cpm}$ (cycles per minute), the values of the Paris constants are: $C=8,84 * 10^{-7}$ cycles $/ \mathrm{mm}$ and $m=1,89$ (Fig. 8). 


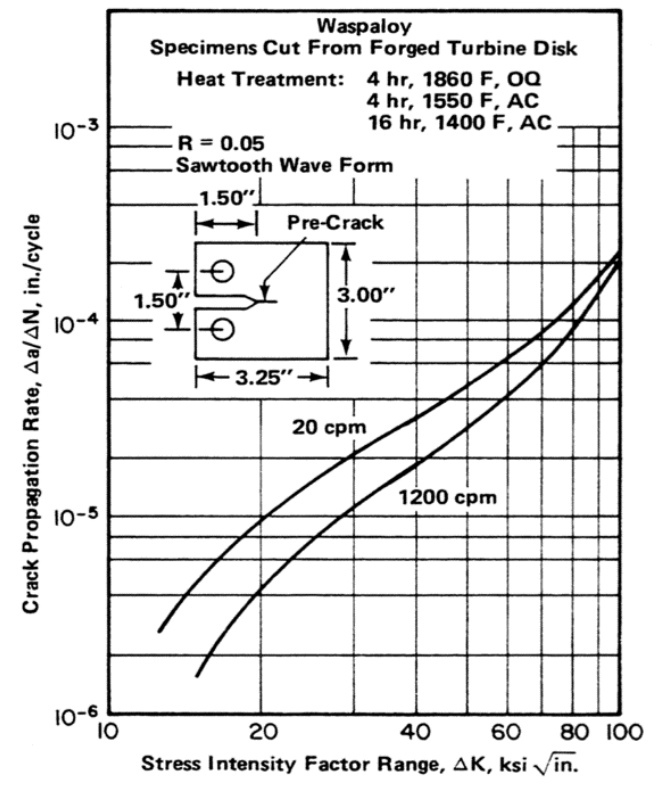

Fig. 8. Plot da/dN in function of $\Delta K$ for the disc material tested in $650^{\circ} \mathrm{C}$ (for specimens cut from the forged turbine disc) [21].

The $d a$ and $d N$ parameters in the Paris-Erdogan solution can be written in the finite (increment) form as:

$$
\frac{\Delta a}{\Delta N}=C \cdot(\Delta K)^{m}
$$

where:

$$
\Delta a=a_{2}-a_{1}
$$

$a_{2}, a_{1}$ - the cracks length between adjacent increments

$\Delta N$ - number of cycles which is needed for growth of the crack from size $a_{1}$ to $a_{2}$.

After transforming the equation (3), the partial number of cycles $\Delta N$ for growth of the crack from size $a_{1}$ to $a_{2}$ can be expressed as follows:

$$
\Delta N=\frac{\Delta a}{C \cdot(\Delta K)^{m}}
$$

The total number of cycles is computed by summing the partial $(\Delta N)$ numbers of cycles:

$$
N=\sum_{i=1}^{n} \Delta N
$$

where: $n$-number of all increments.

Based on the $\Delta \mathrm{K}$ values and Paris-Erdogan equation, the fatigue crack growth curves were determined. The results presented in Fig. 9 show that the crack needs about 7000-8000 cycles of turbine acceleration to grow from $1 \mathrm{~mm}$ to $7 \mathrm{~mm}$. 


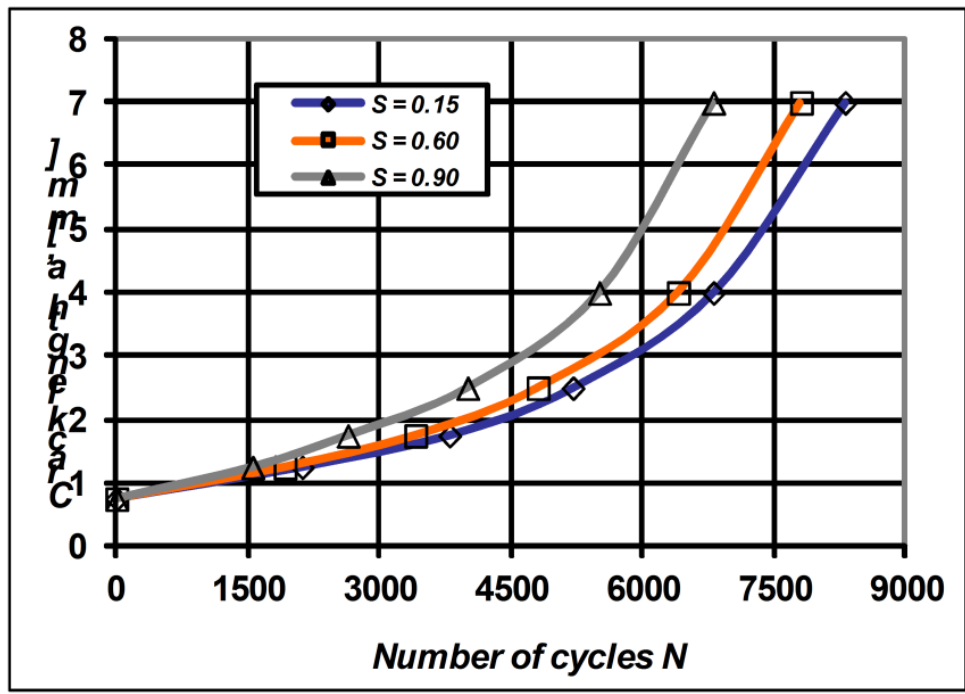

a)

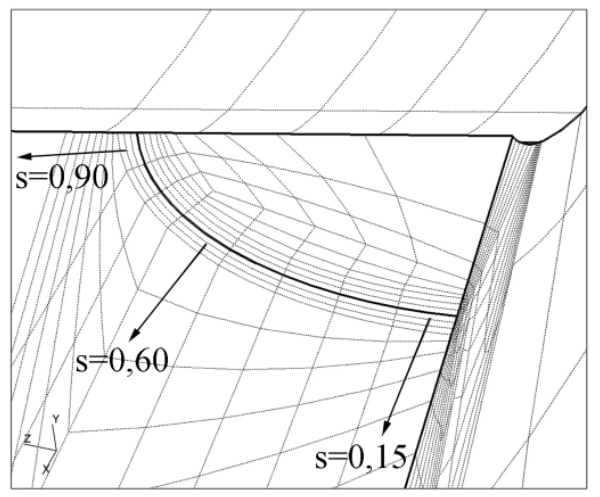

b)

Fig. 9. Crack growth plots obtained for a crack propagated in different directions (a) and the graphical visualization of directions $S=0,15 ; S=0,60$ and $S=0,90$.

\section{CONCLUSIONS}

In this work the crack propagation analysis of the turbine disc subjected to thermo-mechanical forces was performed. In the first part of the work, a complex finite element model of the turbine segment was defined. As a result of nonlinear computations, the stress intensity factor $\left(\mathrm{K}_{\mathrm{I}}\right)$ for the quarter elliptical cracks was obtained. Based on $\Delta \mathrm{K}$ values computed using FEM and the ParisErdogan solution, the crack growth curves were obtained for the disc with the rotation speed oscillating between $6373-14200$ RPM.

The estimation of the K-factor and crack growth in the turbine disc subjected to thermomechanical fatigue is an original research work from the point of view of fracture mechanics and fundamental sciences in general. The results of similar analyses are not widely described in literature because of the complexity of the problem. Information about crack growth has great practical significance and can be useful to determine the periods between technical inspections of the engine. During these inspections, crack should be detected before its growth to the critical size. This kind of operation, known in literature as "damage tolerance" is used for the aircraft and turbine engines. The procedure (damage tolerance) can be used not only in aviation but also in many other branches, for different parts working in variable loads conditions. 


\section{REFERENCES}

[1] Chan S.K, Tuba I.S. (1971). A finite element method for contact problems of solid bodies - Part II: Applications to turbine blade fastenings. International Journal of Mechanical Scientist; 13:627-39.

[2] Masataka, M. (1992). Root and groove contact analysis for steam turbine blades. Japan Society Mech. Eng. Int. J., 35:508-14.

[3] Meguid S.A, Kanth P.S, Czekanski A. (2000). Finite element analysis of fir-tree region in turbine disc. Finite Element in Analysis and Design, 35:305-17.

[4] Papanikos P, Meguid S.A, Stjepanovic Z. (1998). Three-dimensional nonlinear finite element analysis of dovetail joints in aero-engine discs. Finite Element in Analysis and Design, 29:17386.

[5] Zboinski, G. (1995). Physical and geometrical non-linearities in contact problems of elastic turbine blade attachments. J Mech. Eng. Sci., 209:273-86.

[6] McEvily, A. (2004). Failures in inspection procedures: case studies. Engineering Failure Analysis, 11:167-76.

[7] Barlow K.W., Chandra R. (2005). Fatigue crack propagation simulation in an aircraft engine fan blade attachment. International Journal of Fatigue, 27:1661-1668.

[8] Slavik D., McClain R., Levis K. (2000). Stress intensity predictions with ANSYS for use in Aircraft engine component life prediction. Fatigue and Fracture Mechanics, Vol. 31(2000), 371390.

[9] Zhuang, W. Z. (2000). Prediction of crack growth from bolt holes in a disc. International Journal of Fatigue, Vol. 22(2000), p. 241-250.

[10] Shlyannikov V., Iltchenko B., Stepanov N. (2001) Fracture analysis of turbine disks and computational-experimental background of the operational decisions. Engineering Failure Analysis, Vol. 8(2001), p. 461-475.

[11] Witek, L., Failure analysis of turbine disc of an aero engine, Engineering Failure Analysis, Vol: 13 Issue: 1, Elsevier, 2006.

[12] Witek, L. Experimental crack propagation and failure analysis of the first stage compressor blade subjected to vibration, Engineering Failure Analysis Vol. 16, Issue: 7, Elsevier, 2009.

[13] Witek, L. Experimental crack propagation and failure analysis of the first stage compressor blade subjected to vibration, Engineering Failure Analysis Vol. 16, Issue: 7, Elsevier, 2009.

[14] Witek, L. (2011) Crack propagation analysis of mechanically damaged compressor blades subjected to high cycle fatigue. Engineering Failure Analysis, Vol. 18(4), Elsevier Science.

[15] Witek L., Orkisz, M.. Wygonik P., Musili D. N., Kowalski T. (2011). Fracture analysis of a turbine casing. Engineering Failure Analysis, Vol. 18(3), Elsevier Science.

[16] Witek L., Numerical stress and crack initiation analysis of the compressor blades after foreign object damage subjected to high-cycle fatigue, Engineering Failure Analysis, Volume: 11, Issue: 8, Elsevier Science, 2011.

[17] Witek, L. (2011). Stress intensity factor calculations for the compressor blade with half-elliptical surface crack using Raju-Newman solution. Fatigue of Aircraft Structures, Issue 2011. Institute of Aviation Scientific Publications, Warsaw, Poland.

[18] MSC-PATRAN User's manual, MSC Corporation, Los Angeles; 2009.

[19] ABAQUS User's Manual, ver. 6.8, Abaqus Inc.; 2009.

[20] Anderson T. L., Fracture mechanics. Fundamentals and applications, CRC Press Inc, Boca Raton, Florida, 1991.

[21] Aerospace Structural Metals Handbook, US Air Force CRDA/Purdue University, $37^{\text {th }}$ Edition, vol. 5, 2004. 